Meta

Journal des traducteurs

Translators' Journal

\title{
Le vocabulaire des transformateurs
}

\section{Marcel Longpré}

Volume 14, numéro 4, décembre 1969

URI : https://id.erudit.org/iderudit/003514ar

DOI : https://doi.org/10.7202/003514ar

Aller au sommaire du numéro

Éditeur(s)

Les Presses de l'Université de Montréal

ISSN

0026-0452 (imprimé)

1492-1421 (numérique)

Découvrir la revue

Citer cet article

Longpré, M. (1969). Le vocabulaire des transformateurs. Meta, 14(4), 208-211. https://doi.org/10.7202/003514ar d'utilisation que vous pouvez consulter en ligne.

https://apropos.erudit.org/fr/usagers/politique-dutilisation/ 


\section{LE VOCABULAIRE DES TRANSFORMATEURS}

Le vocabulaire est un ensemble de termes propres à un domaine plus ou moins étendu, accompagnés ou non de leurs définitions. Le présent article n'est pas un vocabulaire mais plutôt une série d'observations préparées à la suite de la lecture du vocabulaire et de quelques documents de la Commission électrotechnique internationale (C.E.I.).

1. Cf. Paul Robert, Dictionnaire alphabétique et analogique de la langue française, Paris, Société du Nouveau Littré, 1965, vol. II, p. 1664.

2. "Toute la seconde partie du film est conçue suivant le procédé traditionnel du montage à deux temps, menant parallèlement deux actions qui se rejoignent à la fin. " (Georges Charensol, Nouvelles littéraires, 17 novembre 1960).

3. Cf. Paul Robert, op. cit. 
La C.E.I. a préparé un vocabulaire électrotechnique dont la deuxième édition comporte une vingtaine de sections. La section 10 , intitulée «machines et transformateurs » date de 1954. Une troisième édition est en préparation et, dans cette nouvelle édition, une section entière sera consacrée aux transformateurs.

Le transformateur joue un rôle important dans le transport de l'énergie électrique. C'est lui qui permet d'élever la tension au départ des grandes lignes de transport et de l'abaisser à l'arrivée aux points d'alimentation.

Un transformateur de puissance (power transformer) ou transformateur est un appareil statique destiné à transformer un système de courants alternatifs en un autre système de même fréquence et d'intensité et de tension différentes. L'auto-transformateur (auto-transformer) est un transformateur dans lequel deux enroulements ont une partic commune. On dit d'un transformateur qu'il a une puissance nominale (rated power, rated capacity) de ...MVA et non une "capacité ». En général, la capacité est la propriété d'un récipient de contenir une certaine quantité de quelque chose. Le litre, la pinte, le baril sont des mesures de capacité. En électrotechnique, la capacité (capacitance) d'un conducteur est le quotient de la charge du conducteur isolé par son potentiel. La capacité se mesure en farads.

Construction - Le transformateur est habituellement immergé (immersed) dans un diélectrique liquide (insulating liquid). L'expression « à bain d'huile 》 s'applique plutôt aux disjoncteurs. Quand il n'est pas immergé dans un diélectrique liquide, c'est un transformateur sec (dry-type transformer). L'huile minérale (mineral oil) est le diélectrique liquide le plus utilisé, mais l'askarel (askarel), produit synthétique ininflammable, sert dans les installations intérieures. Dans le commerce, l'askarel est connu sous les noms de pyralène, pyranol, Inerteen. Il est à noter cependant qu'en France, le mot pyralène se répand de plus en plus pour désigner une certaine catégorie d'askarels.

Le transformateur est essentiellement constitué d'un noyau magnétique (magnetic core) entouré d'un, de deux ou de plusieurs enroulements (windings). Selon la forme du noyau, on distingue les transformateurs à colonnes (core type transformer) et les transformateurs cuirassés (shell type transformer). Les enroulements sont reliés à des conducteurs extérieurs par des pièces conductrices appelées bornes (terminals). La traversée (bushing) permet au conducteur de passer de l'intérieur à l'extérieur de la cuve tout en demeurant isolé. Les mots borne et traversée sont souvent confondus. On prend alors la partie pour le tout. En effet, la traversée comporte une partie en porcelaine à l'intérieur de la cuve (tank), une autre à l'extérieur, un conducteur et, au bout du conducteur, une borne.

Un conservateur (expansion tank) permet d'assurer la dilatation de l'huile. Cette huile est en communication avec l'air extérieur par un respirateur (breather). Le respirateur est souvent complété par un assécheur (dehydrator) qui contient habituellement du gel de silice (silica-gel).

Une soupape de sûreté (explosion vent) protège le transformateur contre les surpressions internes. Elles comporte la plupart du temps une tubulure de 
déversement (explosion vent pipe) pour éjecter l'huile hors du couvercle (cover) et des bornes (terminals).

Enroulements - Un enroulement (winding) ou bobinage est un ensemble de spires (turns) formant un circuit électrique associé à l'une des tensions pour lesquelles le transformateur a été établi. Primaire (primary), secondaire (secondary) et tertiaire (tertiary) sont des qualificatifs qui désignent les enroulements. Selon la façon de connecter les enroulements, on peut distinguer la connexion étoile (star connection), la connexion triangle (delta connection) et la connexion en zigzag (zigzag ou interconnected star connection).

Prises - Les enroulements de transformateurs peuvent être munis de prises (taps) permettant de modifier le rapport de transformation (voltage ratio) par ajustement du nombre de spires en circuit. Le mécanisme de commande qui doit être mancuvré (operated) hors tension est un ajusteur de rapport (ratio adjuster). Celui que l'on peut manœuvrer sur l'appareil en charge est un changeur de prises en charge (on-load tap changer). Le changeur de prise comprend un mécanisme d'entraînement (driving mechanism), un commutateur (diverter switch), et autres accessoires.

Le passage d'une prise à une autre peut être précisé par les mots augmente (raise) et diminue (lower) quand le rapport de transformation augmente ou diminue. Les mots « hausse ». et «baisse » utilisés au Québec sont des anglicismes.

Refroidissement - L'évacuation de la chaleur provenant des pertes peut s'effectuer par contact direct entre le fluide de refroidissement et les organes actifs ou par l'intermédiaire du diélectrique liquide dans lequel ils sont immergés. Le transformateur qui ne comporte ni pompe (pump) ni ventilateurs (cooling fans) est à refroidissement naturel (self-cooled). L'emploi de l'adjectif « autorefroidi » pour qualifier ce mode de refroidissement est un anglicisme.

Pertes - Les pertes (losses) sont la différence entre la puissance qui est fournie au transformateur et celle qui est restituée par celui-ci. Les pertes habituellement mesurées sont les pertes à vide (no load loss) et les pertes dues à la charge (load loss).

Essais - Les essais (tests) effectués sur les transformateurs ont pour but de montrer que ceux-ci satisfont aux conditions spécifiées. Les essais de type (type tests) ne sont effectués que sur les appareils représentatifs d'une fabrication; les essais individuels (routine tests) sont effectués sur chaque transformateur. Les principaux essais sont la mesure du rapport de transformation (voltage ratio measurement), la mesure des tensions de court-circuit (measurement of impedance voltages), les essais d'échauffement (tests of temperature rise), les essais de tenue au choc (impulse-voltage withstand test).

Quand les essais de réception (acceptance tests) ont prouvé à l'acheteur que le transformateur est conforme aux spécifications, cet acheteur signifie son acceptation (acceptance). Le contraire serait le rejet (rejection). La confusion entre les mots réception et acceptation est due à l'influence de l'anglais. 
Transformateurs de mesure - Un transformateur de mesure (instrument transformer) est celui qui est destiné à alimenter des appareils de mesure, des compteurs, des relais et autres appareils analogues.

Le transformateur de courant (current transformer) et le transformateur de tension (voltage ou potential transformer) sont des transformateurs de mesure qui, quand ils sont montés dans une même cuve, prennent le nom de combiné de mesure (metering unit ou outfit).

La normalisation du vocabulaire électrotechnique par la Commission électrotechnique internationale est en bonne voie. La participation d'un grand nombre de pays et la diffusion de plus en plus importante de ses documents sont des signes encourageants. Souhaitons qu'en plus de l'unification des termes nous en arrivions à l'unification des notions ou définitions.

MARCEL LONGPRÉ 